\title{
Entrelacs
}

Cinéma et audiovisuel

Nouvelles formes audiovisuelles documentaires

\section{Processus schizophrénique augmenté, ou quand le web s'en mêle, retour sur Journal d'un coopérant, projet interactif de Robert Morin}

\section{Caroline San Martin}

\section{(2) OpenEdition \\ Journals}

Electronic version

URL: http://journals.openedition.org/entrelacs/1880

DOI: $10.4000 /$ entrelacs. 1880

ISSN: 2261-5482

Publisher

Éditions Téraèdre

\section{Electronic reference}

Caroline San Martin, «Processus schizophrénique augmenté, ou quand le web s'en mêle, retour sur Journal d'un coopérant, projet interactif de Robert Morin », Entrelacs [Online], 12 | 2016, Online since 14 January 2016, connection on 20 April 2019. URL : http://journals.openedition.org/entrelacs/1880 ; DOI : 10.4000/entrelacs.1880

This text was automatically generated on 20 April 2019.

Tous droits réservés 


\title{
Processus schizophrénique augmenté, ou quand le web s'en mêle, retour sur Journal d'un coopérant, projet interactif de Robert Morin
}

\author{
Caroline San Martin
}

Robert Morin est un fabulateur, un cinéaste qui se légende lui-même.

\begin{abstract}
Mais remarquez qu'il y a trois choses qui m'intéressent, explique-t-il, trois veines à explorer : ce genre de film avec du "vrai monde" (Quiconque meurt..., Toi t'es-tu lucké), les petits films de fiction, à petits budgets, avec un "je" plus directement connecté ( Gus est encore dans l'armée, Yes Sir! Madame..., Le Voleur vit en enfer), puis le long métrage de fiction, la grosse bébelle ${ }^{1}$.
\end{abstract}

2 Ce sont précisément ces films au « je » qui vont nous intéresser. Le réalisateur y incarne des personnages-filmeurs se mettant en scène, changeant plus ou moins son identité. Tantôt politique, dans Yes Sir ! Madame... (1994) ; chômeur, dans Le Voleur vit en enfer (1984) ou pseudo altermondialiste en Afrique pour Journal d'un coopérant (2010), il essaie des rôles à partir d'une même image, la sienne.

Pour ma santé mentale, il est important que je tourne, fait-il remarquer. Peu importe qu'il y ait de l'argent ou non. C'est sûr, j'aimerais être dans le système des fois, mais je n'ai pas envie d'un cinéma formaté. J'aime essayer des affaires. J'aime fouiller le "je", faire des films à la première personne du singulier. Souvent, le cinéma, ce n'est que du "il", c'est-à-dire la vision d'un auteur qui se met à distance sans se mouiller. La plupart du temps, on est dans la déclamation : du gros $35 \mathrm{~mm}$, de la grosse image, de gros effets, de gros personnages. Je préfère me rapprocher un peu. Créer une communication plus intime avec le spectateur. Quitte à le trahir ${ }^{2}$ !

Tous ces films au «je » mettent en scène une trahison parce que les personnages sont toujours plus complexes que ce que nous croyons et tous posent la question du double, de l'invention d'un autre à travers la représentation du même. Les mises en abymes se succèdent encourageant une libre circulation entre l'intra et l'extra-diégétique : celui qui filme dans la fiction est le même que celui qui filme dans le réel. Dans Journal d'un 
coopérant, le processus se complexifie. En 2006, Robert Morin rejoint sa compagne au Rwanda sur le tournage du film québécois Un dimanche à Kigali de Robert Favreau ${ }^{3}$.

Là, explique Robert Morin :

j'ai vu les rutilants $4 \times 4$ blancs de l'ONU, les inégalités sociales et la division des classes. J'ai aussi vu avec qui les hommes se tiennent dans leurs moments de loisir. C'est de là qu'est née l'idée du film. J'ai alors fait des recherches sur la coopération internationale. Tous les chiffres avancés dans le film sont véridiques. En abordant un sujet comme celui-là, il est important de faire ses devoirs ${ }^{4}$.

Le personnage qu'il interprète, Jean-Marc Phaneuf, est un électronicien célibataire qui se rend à Ujama, en Afrique, à titre de coopérant pour l'ONG "Radio du Monde". Il découvre un pays accablé par la pauvreté, la famine, la guerre, la maladie et une scandaleuse inégalité sociale. Parallèlement, il y fait la rencontre d'un peuple joyeux, courageux, assoiffé de bonheur, de savoir et de dignité humaine. La caméra lui sert de journal intime et va progressivement lui permettre de dévoiler les rouages douteux et inefficaces des ONG. Bien qu'elle souligne certains efforts louables de la coopération internationale, l'investigation de Jean-Marc débouche sur un constat terrible : l'aide humanitaire est une illusion, une utopie ${ }^{5}$.

6 A première vue, la caméra produit les mêmes effets que dans les films précédents. Les images que nous voyons sont celles tournées par le personnage (il y a un second opérateur, ce qui permet plus de possibilités de montage, mais, d'un point de vue diégétique, ce dernier n'existe pas). Souvent à l'aide de miroirs, le personnage-filmeur est mis en scène et signifié comme le producteur des images projetées sur l'écran (figure 1).

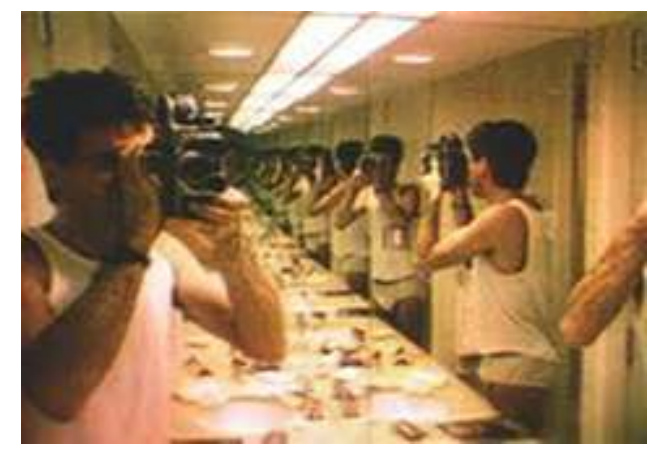

Figure 1. Journal d'un coopérant : le personnage actionne une télécommande pour lancer ou stopper l'enregistrement

7 Sa voix les commente telle une voix-off de documentaire, mais elle fait aussi de temps en temps penser à celle du réalisateur qui dirige ses acteurs - il dit aux personnages quoi dire et comment se positionner devant la caméra. Pourtant, cette voix est bel et bien in puisque simultanée de la prise d'images et appartenant au même espace diégétique. Le personnage est double. Mais la démultiplication est ici exponentielle : le filmeur est aussi blogueur. Journal d'un coopérant est le premier film québécois issu du Web 2.0. Du 3 décembre 2009 au 29 janvier 2010, sous la forme d'un blog, les vidéos du film en devenir sont diffusées telles un journal de bord (figure 2). 


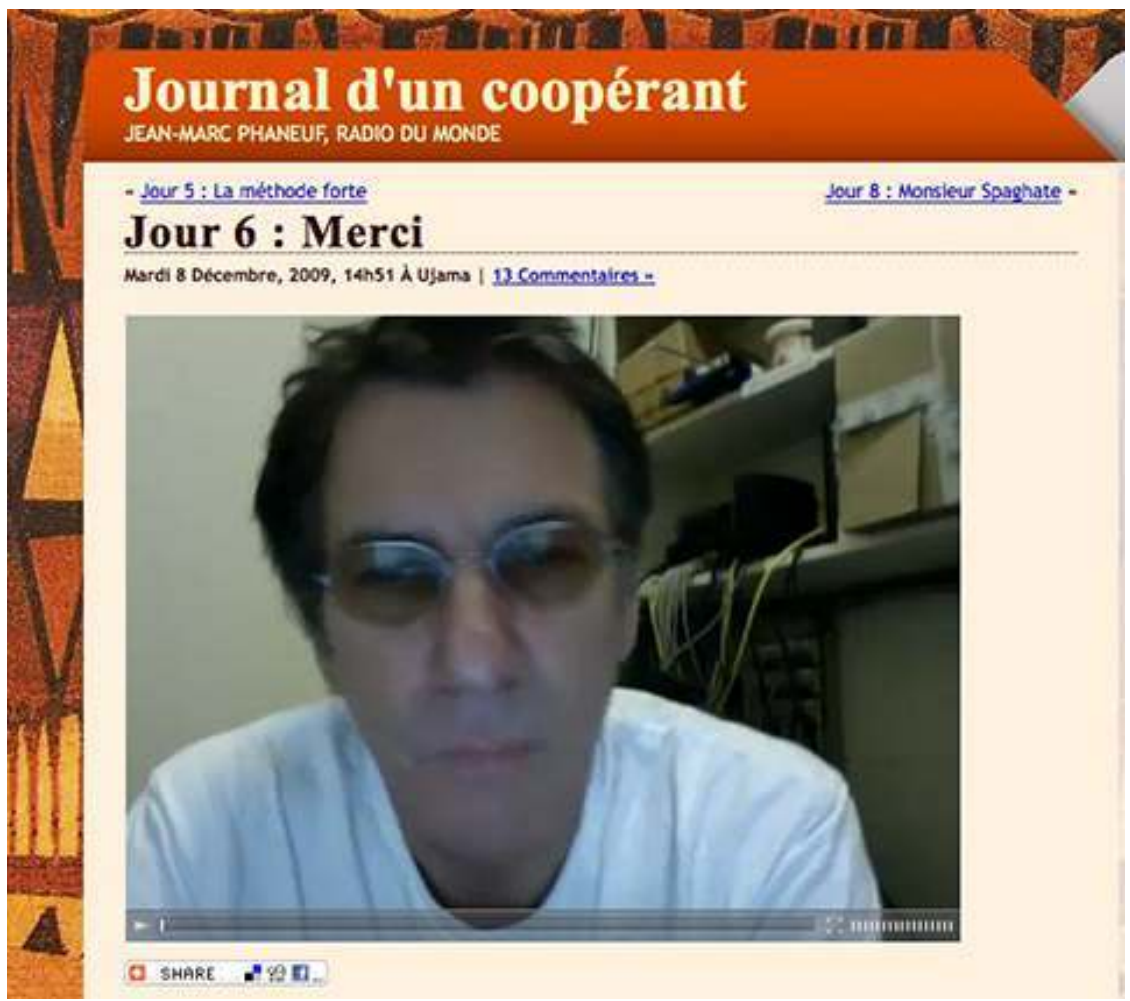

Figure 2. Yes Sir! Madame... Earl Tremblay dans sa salle de bain

C'est une expérience que je voulais tenter, explique le réalisateur. Même si j'aurais aimé que les gens s'expriment davantage par webcam, ce fut très positif. Rarement peut-on s'offrir le luxe de décanter un projet pendant deux mois et de l'enrichir au gré des observations que des intervenants peuvent faire. Cela dit, la trame du scénario était fixée depuis le début. Je savais très bien où je m'en allais ${ }^{6}$.

D'abord conçue comme une plateforme participative, le réalisateur a proposé aux internautes de suivre cette expérience interactive quotidiennement. Beaucoup de blogs ont d'ailleurs permis de médiatiser l'entreprise sur la toile, comme celui de l'écrivain Mathieu Arsenault, doctorak.go, qui explique le projet et accompagne les internautes dans l'expérience proposée par le cinéaste.

S'il arrivait que vous ne croyiez plus comme moi à l'industrie du cinéma, vous pourriez considérer la sortie du film en salles comme une publication posthume du blog destinée aux archives. Car ce dispositif narratif que Morin a développé depuis ses premières vidéos, qui fait s'entrecroiser fiction et réalité, s'adapte parfaitement à la forme du blog. Le site met beaucoup l'accent sur le système des commentaires et vous êtes cordialement invités à embarquer dans la fiction de Morin, à inventer un personnage qui existe sur le même plan de réalité que Jean-Marc Phaneuf. Ainsi, sa sœur, ses amis et tout un univers de personnages imaginés par les commentateurs meublent peu à peu l'espace du blog. Et il arrive un moment où le dispositif de Morin se met à fonctionner parfaitement et on ne sait plus si les lecteurs savent ou non si le blog est fictionnel ou autobiographique ${ }^{7}$.

Si le regard du cinéaste se confond avec celui du personnage qu'il crée, il propose aux blogueurs d'adopter la même position que lui et de fabuler avec lui autour de la fiction qu'il est en train d'inventer. Si bien que la toile s'affirme comme un espace d'imaginaire collectif autour d'images prises dans le réel. Cette position continue de faire croître le nombre des rôles endossés par un corps : ils sont désormais trois personnages en un. Mais ce nombre augmente encore. Au milieu du film, Jean-Marc, vivant seul, s'ennuyant 
pendant ses longues soirées africaines, commence à jouer en ligne en s'inventant un avatar. Robert Morin exploite les médias comme un moyen d'expression et non comme un processus technique, et laisse entrevoir de nouvelles possibilités créatrices. Ce film continue de poser les problèmes soulevés par son cinéma à travers une réflexion sur le personnage en y intégrant le web, encourageant des processus schizophréniques à travers la démultiplication des identités physiques et numériques.

Tous les films au « je » de Robert Morin jouent sur ce terrain. Les personnages ne sont pas construits autour d'un :

"je suis ceci, je suis cela" [...] il faut penser en termes incertains, improbables : je ne sais pas ce que je suis, tant de recherches ou d'essais nécessaires, non-narcissiques, non-œdipiens [...]. Le problème n'est pas celui d'être ceci ou cela dans l'homme, mais plutôt d'un devenir inhumain, d'un devenir universel, animal : non pas se prendre pour une bête, mais défaire l'organisation humaine du corps, traverser telle ou telle zone d'intensité du corps, chacun découvrant les zones qui sont les siennes, et les groupes, les populations, les espèces qui les habitent ${ }^{8}$.

11 Et c'est cela que permet cette démultiplication chez Morin. Revenons à Yes sir, Madame... Voici ce qu'inscrit le dossier de presse quant au résumé du film. "Né d'un père francophone et d'une mère anglophone, Earl Tremblay vit une crise d'identité. Une crise qu'il essaiera de régler en fouillant son passé, son présent et même son futur, au moyen d'une caméra ${ }^{9}$. » Film résolument politique, la crise identitaire est bien au centre de ce dernier : il ne s'agit pas tant de faire que de défaire, de revoir pour voir autrement. «It's gonna make a fucking good Canadian movie, ouais, ça va faire un criss de bon film canadien », nous livre Earl Tremblay post-générique. «Il est évident qu'une partie de la problématique du Canada demeure sans contredit la présence dominante de nos voisins du Sud, explique Robert Morin. A partir de cette constatation, il parait difficile de conserver une langue ou une culture ${ }^{10} \%$. Le réalisateur québécois scinde son film, il en sépare tous les éléments: Earl est un canadien qui parle français et anglais, il s'autotraduit à chaque phrase. Il met en scène la dimension plurielle de l'être, métonymie pour le pays dans lequel il vit, et ce, à travers la dimension plurielle du médium cinématographique. Le son et l'image sont aussi clairement dissociés. Par conséquent, dans les programmes qui proposent le film, le descriptif en révèle la complexité. Le communiqué de Art Metropole à Toronto qualifie l'œuvre comme étant un "vidéogramme, couleur et noir et blanc, bilingue" rejouant ainsi la complexité identitaire du pays dont il est issu. Le dispositif est simple, un homme dont nous voyons peu le visage, projette sur un écran, qui nous est donné comme cadre, des images tournées en 16 millimètres. Il s'agit d'un député dont nous découvrons à titre posthume un enregistrement. Earl est dans son salon et nous explique dans deux langues qu'il ne se souvient pas de ce qu'il a filmé sur les deux dernières bobines; bobines, qu'il commente au fur et à mesure. Le film commence par une mise en situation : le jour de sa mort, sa mère lui avait donné une caméra ainsi que 19 bobines. « Her death wish was for me to use the camera to find myself ${ }^{1{ }}$ ». C'est littéralement ce qu'il va faire : ce n'est pas lui mais son image - un double de lui-même - qui lui permet d'avoir accès à la complexité de ce qu'il est.

Dans Journal d'un coopérant, la caméra a la même visée. Elle révèle une vision et donne accès au personnage à travers son image et son regard, reposant la question $\mathrm{du}$ « je ».

Depuis Griffith, le cinéma, à quelques exceptions près, évolue dans le "il", ou si vous préférez l'approche objective. Nous voyons les personnages, mais ceux-ci ne voient 
pas. Ils subissent les conséquences de la caméra qui les filme. Par une sorte de détournement, j'essaie de montrer les actions à travers les yeux d'un personnage ${ }^{12}$.

Et ce détournement est incarné par le personnage-filmeur. Ce dernier se découvre en même temps que le spectateur le voit agir. L'audience prend part au film parce qu'elle suit ce «je » en train d'apparaître. Comme dans Yes Sir, il s'agit de mettre en avant une division, une séparation, entre les pays du nord et les pays du sud; les anciens colons et ceux que l'on continue de coloniser. Le film traite donc de la coprésence du double, d'un double antinomique dont la coexistence est problématique voire impossible. Et ce double est incarné par le personnage. Encore une fois, le propos du film se rejoue dans la forme, à l'image de la confusion des genres documentaire et fiction. A priori exclusifs, les films de Morin affirment la porosité des limites permettant une circulation incessante entre le réel et l'imaginaire en considérant la technique et le dispositif. Dans Yes sir! Madame..., il était question de l'espace de projection et du super 16 qui, de part ses caractéristiques techniques, ne peut contenir que des informations visuelles (la bande est trop mince pour qu'on inscrive, de surcroit, des informations sonores). De ce fait, le narrateur, clairement identifié comme omniscient, nous raconte les histoires de ces images, leurs contenus dramatiques, en habitant les corps des différents protagonistes présents dans le cadre grâce à un petit dictaphone. Dans Journal d'un coopérant, le format de l'écran rappelle souvent celui des fenêtres diminuées sur Internet et la multiplication des rôles incarnés par Jean-Marc reflète la multiplicité de nos identités et la façon dont nos vies deviennent des espaces de représentation. C'est pourquoi le film va reposer sur des systèmes d'échos. Il met en avant une esthétique du fragment. L'image devient un fragment dans l'espace de projection qu'est l'écran. Située au centre, à plusieurs reprises, le réalisateur s'amuse avec la référentialité, rappelant le lieu dont le film est issu - le web. L'image épouse le format de celles que nous pouvons retrouver sur youtube, elle n'occupe pas tout le cadre. Elle peut s'agrandir progressivement décomposant ainsi l'instantané du clic lorsque la souris vient activer l'icône permettant à la fenêtre d'épouser la taille de l'ordinateur. D'autres fois, cet écran est explicitement présent à l'image, notamment quand l'avatar du réalisateur crée lui-même un autre avatar dans un jeu de rôle en ligne (figure 3).

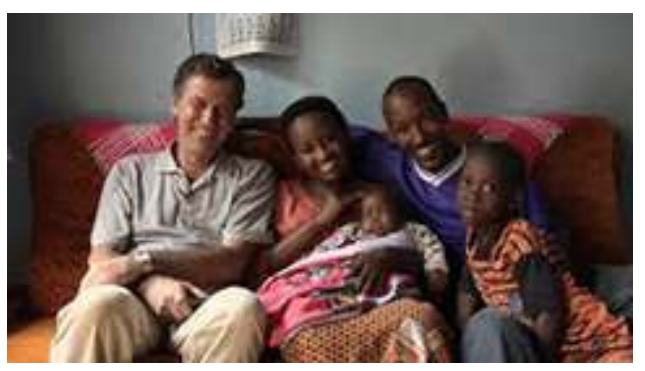

Figure 3. Journal d'un coopérant : le blog

C'est alors que l'écran de l'ordinateur filmé devient le cadre de l'image rejouant la mise en abyme portée par le personnage : le réalisateur joue le rôle d'un coopérant qui filme tout ce qui l'entoure, lui-même réalisateur de son journal de bord. Cette esthétique du fragment peut aussi être considérée comme inhérente au genre - le journal de bord. Le principe même de l'écriture repose sur des extraits de journées dont la successivité des dates permet de combler les ellipses. Malgré quelques raccords qui masquent le fait de tourner à deux caméras et donnent l'illusion de plans-séquences, notamment lors des mouvements d'accompagnement quand le protagoniste suit en caméra épaule un personnage lui permettant de découvrir un lieu (la station de radio par exemple), les 
raccords sont toujours dans le mouvement, sans réduction d'échelle de plan ou changement d'axe permettant de donner la sensation que le temps s'étire. Ces durées de plans, réelles (il y a aussi de longs plans-séquences) ou artificielles (construites par les raccords) contrastent avec les multiples plans de coupes donnant à voir quelques images des villes traversés par Jean-Marc comme le ferait une série de cartes postales. Ces plans traduisent bien le survol furtif de l'occidental et l'exotisme de l'Afrique anticipant la question, bien avant la résolution du film, de l'ambiguïté du regard du personnage. En effet, même si le dispositif reste similaire avec celui de Yes Sir, le problème se déplace. Contrairement à Earl, Jean-Marc n'est pas amnésique et la question n'est plus la crise de l'identité mais la complexité d'un regard et des lieux de représentation. Le film affirme bien que nos perceptions ne sont jamais neutres et si la réalité se confond avec une carte postale et l'exotisme des paysages est fait pour séduire, c'est la même démarche qui est mise en avant quand Jean-Marc filme Mathilde, la fille de sa femme de ménage. Elle apparaît comme une magnifique jeune fille noire. Avant même la connotation sexuelle de ce regard, quelque chose sonne faux: peut-on filmer des corps étrangers comme des paysages exotiques de cartes postales? Les corps et les paysages sont-ils uniquement là pour séduire l'œil ? L'ambiguïté et la réduction de ce regard résonnent avec l'exposition Fucking Tourist (2012) ${ }^{13}$ du photographe Nicolas Demeersman (alias Jolipunk) où, pour manifester leur mécontentement face au fait d'être mitraillés par les touristes et réduits à des objets folkloriques, cadrés en portraits, des Cubains, des Amérindiens, des Péruviens ou des Indiens brandissent un doigt d'honneur face à l'objectif. Pourtant, au milieu du film, Jean-Marc est le seul occidental à voir l'attitude ambigüe des institutions humanitaires et à interroger leur vision post-colonialiste : les coopérants ont des maisons avec piscines, un garde pour ouvrir leur portail, une femme de ménage pour leur faire à manger, etc. Il est le seul personnage blanc critique - Léopold (son traducteur) explique autour d'un repas la répartition de l'aide financière en Afrique, ce qui le scandalise. Pour autant, Jean-Marc, comme Earl, est pris dans un rapport métonymique : il va permettre à Mathilde et à ses amis d'accéder aux richesses réservées aux blancs - les enfants sont autorisés à se baigner dans la piscine - il lui achète des vêtements et un maillot de bain occidentaux, lui apprend à jouer à l'ordinateur. Il colonise ses pratiques sociales (la piscine), son apparence (les vêtements) et son corps (il la couche sur le lit et la déshabille) avec la même autorité, la même fausse générosité et la même violence que les colons blancs occidentaux. "Oui, c'est vrai. Mes personnages sont, pour la plupart, des marginaux parce que ce sont eux qui se questionnent le plus souvent et poussent le reste de la société à s'interroger ${ }^{14}$. " Jean-Marc pose la question de la fausse entraide mondiale à travers celle de la pédophilie.

15 Le film intègre son propre discours critique. Il propose de réfléchir les formes audiovisuelles documentaires et fictionnelles. Le jeu dans le jeu tout comme le « je » dans le «je » anticipent les avancées dramatiques. L'avatar est créé avant le rapprochement avec Mathilde et annonce celui-ci par le biais du jeu de rôle. Le récit dans le film rejoue le dispositif mis en avant par Morin : le blog permet un mouvement de la réalité (le blog était réel, les commentaires se faisaient dans un espace non diégétisé) vers la fiction (ces prélèvements du réel sont agencés pour créer un film). Dans le film, le mouvement est inverse : le jeu, purement virtuel puisque contenu dans l'espace de l'ordinateur, empiète progressivement sur le quotidien des personnages et ne peut plus être confiné dans un seul espace. Le faux baiser entre Jean-Marc et Mathilde à l'intérieur de l'écran dans l'écran se déplace dans la chambre à coucher. Cette multiplication de mises en abyme 
implique forcément le spectateur. André Chastel l'a théorisé en peinture grâce au concept du tableau dans le tableau ${ }^{15}$. Par extension, le cadre dans le cadre au cinéma a toujours été une stratégie pour mettre en avant un discours critique sur la façon de regarder l'image. L'historien d'art explique que le tableau contenu peut redire l'enjeu dramatique du tableau contenant, mais il peut aussi informer sur le fonctionnement de l'œuvre et la façon dont elle doit être regardée. Si bien que, dans le film, même si la multiplicité des supports engage le spectateur, le cadre dans le cadre et la mise en abyme comme procédés stylistiques ne cessent d'annuler sa passivité. Ils modulent, manipulent, informent quant au parcours du regard spectatoriel. Alors nous prenons part au jeu. Puisqu'il s'agit de cela. Le film devient en quelque sorte «interactif » dans le sens où il nous propose les clés pour le lire dans les sous-cadres qu'il produit. Un jeu d'anticipation se met en place. Plus loin que la médiation, le film recherche l'interaction. Cette dernière s'affirme comme puissance, une puissance du faux pour accéder à l'inaudible, pour toucher l'invisible - la scène finale avec Mathilde. Cet excès de représentation des images, ces séries de fonctionnements en poupées russes questionnent finalement notre façon de produire et de recevoir des représentations, d'en amoindrir certaines, de refuser leur violence en nous contentant des cartes postales. «Toucher le spectateur, ça ne veut pas obligatoirement dire le faire pleurer et lui donner un happy end en épilogue. Ça veut aussi dire le déranger, le bousculer, le confronter à des idées reçues. Mon travail, c'est de provoquer, de questionner ${ }^{16}$.» Questionner la représentation du «je », c'est forcément questionner celui qui produit et celui qui perçoit un regard. Le film permet de rendre visible cette alliance virtuelle qui constitue le spectateur (recevoir et produire un message) en lui proposant qu'elle ne reste plus intellectuelle mais devienne pratique par le biais $\mathrm{du}$ web. Le médium participe au propos parce qu'il incarne cette posture spectatorielle et sollicite un engagement.

Je suis très content de cet effet, affirme le cinéaste. J'aime le cinéma qui dérange, qui nous confronte à nos zones d'ombre. J'aime sortir d'un film avec des points d'interrogation dans la face! Quand j'ai vu Lumière silencieuse de Carlos Reygadas, j'en ai eu pour une bonne semaine à me questionner. Même chose pour Ken Park de Larry Clark. Quand on veut faire un cinéma qui questionne, on n'obtient pas toujours les réponses les plus rassurantes. Je préfère de loin poser ces questions, quitte à être mal compris, plutôt que de me vautrer dans des certitudes et faire un film plate ${ }^{17}$ !

Le narrateur devient le discours et la technique en est le mode de dévoilement.

\section{NOTES}

1. Robert Morin, "S'entêter à faire réfléchir, entretien avec Robert Morin ", propos recueillis le 17 mars 1998 par Joel Pomerleau, Nicolas Renaud, Steve Rioux, http:// www.horschamp.qc.ca/9803/emulsion/morin.html

2. Marc-André Lussier, "Journal d'un coopérant de Robert Morin : ainsi sois-je... » in http://www.lapresse.ca/cinema/nouvelles/entrevues/201207/17/01-4549343-journal- 
dun-cooperant-de-robert-morin-ainsi-sois-je.php, publié le 20 mars 2010 à 12h35, mis à jour le 20 mars 2010 à 12h35, consulté le 26 octobre 2014

3. Robert Morin propos recueillis par Marc-André Lussier in « Journal d'un coopérant de Robert Morin: ainsi sois-je... »

4. Ibid.

5. Ce résumé reprend celui qui figure sur la fiche du film sur le site africultures http:// www.africultures.com/php/index.php?nav=film\&no=11239, consulté le 10 juin 2015.

6. Robert Morin propos recueillis par Marc-André Lussier in «Journal d'un coopérant de Robert Morin: ainsi sois-je... »

7. http://doctorak-go.blogspot.fr/2009/12/vous-voulez-voir-le-prochain-film-de.html, publié le 12 décembre 2009.

8. Gilles Deleuze, «Lettre à un critique sévère » in Pourparlers, éditions de Minuit, 2002, p. 21-22

9. Dossier de presse du film Yes sir ! Madame... de Robert Morin, Québec, 1994.

10. Robert Morin, Séquences, $n^{\circ} 175$, novembre/décembre 1994, p. 16.

11. «Son dernier souhait était que j'utilise cette caméra afin de me trouver moi-même » (ma trad.).

12. Robert Morin, Séquences, $n^{\circ} 175$, novembre/décembre 1994, p. 14.

13. Voilà comment Nicolas Demeersman explique son projet sur son blog: «C'est en photographiant les locaux que l'un d'eux me fit cette remarque : "Désolé, mais tous les touristes nous mitraillent comme des objets de folklore... Et un jour on se retrouve en carte postale ! Donc pas de photo pour moi !".

Et puis je me suis dit : il a raison... faisons des portraits, qui, pour une fois, pourront exprimer leur ressenti : "Fucking tourist !" » http://jolipunk.over-blog.com/articlefucking-tourist_inedits-105167141.html, consulté le 12 mars 2013.

14. Robert Morin, Séquences, op.cit., p. 15.

15. André Chastel, Le Tableau dans le tableau, Fammarion, 2012

16. Robert Morin propos recueillis par Marc-André Lussier in «Journal d'un coopérant de Robert Morin : ainsi sois-je... »

17. Ibid. «Plate » est un québéquisme qui signifie « ennuyeux ».

\section{ABSTRACTS}

Journal d'un coopérant is the first Quebecer movie that came out of the Web 2.0. From December 3rd 2009 and January 29th 2010 as a blog, videos of the future film - released two months later have been posted. It was conceived at first as a participative platform. The director proposed the users to follow his videos daily. He plays the main character, called Jean-Marc Phaneuf. If both visions are melting - the director's and the character's -, he proposes the users to melt reality and fiction as well by inventing their comments in order to fit the fictional universe of Jean-Marc 
Phaneuf so that the web can reveal itself as being a common space of imagination relying on real images.

Journal d'un coopérant est le premier film québécois issu du Web 2.0. Du 3 décembre 2009 au 29 janvier 2010, sous la forme d'un blog, les vidéos du film en devenir sont diffusées telles un journal de bord. D'abord conçue comme une plateforme participative, le réalisateur a proposé aux internautes de suivre cette expérience quotidiennement. Le film, d'une durée d'une heure trente, sort le 26 mars. Le personnage principal Jean-Marc Phaneuf est interprété par le réalisateur québécois. Si le regard du cinéaste se confond avec celui du personnage qu'il crée, il propose aux blogueurs d'adopter la même position que lui et de fabuler avec lui autour de la fiction qu'il est en train d'inventer. Si bien que la toile s'affirme comme un espace d'imaginaire collectif autour d'images prises dans le réel.

\section{AUTHOR}

\section{CAROLINE SAN MARTIN}

Titulaire d'un doctorat en études cinématographiques de l'Université de Provence et d'un doctorat en littérature comparée de l'Université de Montréal, établissements dans lesquels elle a enseigné. Elle est actuellement ATER à l'université Paul Valéry à Montpellier dans le département de cinéma et est rattachée au laboratoire de recherche RIRRA 21. Ses travaux portent l'analyse figurative à travers l'étude de monographies ainsi que sur la façon dont le cinéma, en dialogue avec les autres arts, questionne la représentation. 